\title{
When to restart breastfeeding in a lactating mother after a snakebite?
}

\author{
Maryam Vasheghani-Farahani ${ }^{1}$ and Sayed Mahdi Marashi ${ }^{2}$ \\ AJA university of Medical Sciences, Tehran ${ }^{1}$, Shiraz University of Medical Sciences, Shiraz ${ }^{2}$, Iran
}

Snake bites are frequently reported in both rural and suburban areas worldwide (1). They are especially common in areas with climate and ecological conditions suitable for poisonous snake species. Among the victims of poisonous snakebites, lactating women are no exception. In such cases, one of the most important questions facing a clinical toxicologist is 'when can a lactating patient restart breastfeeding after a poisonous snakebite'. Reviewing the literature, we did not find any suggestion whether mothers who experienced a poisonous snakebite can generally continue breastfeeding or not.

As is known, snake venoms contain various types of proteins, enzymes, lipids, carbohydrates, and metal ions (2). However, it is not known whether these ingredients transfer into a mother's breast milk. Furthermore, even if some of the venom's compounds become ingredients in the bitten mother's milk, it is unclear how the breastfed child's organism would react. An interesting article by Dupre et al. (3), reporting new information on quantifying protein toxins in human biofluids, suggested that protein toxin degradation does not occur in breast milk. Therefore, we can assert that the venom's protein compounds would very likely reach the child's organism. However, considering that certain traditional beverages containing snake venom are consumed, e.g. Chinese snake wine (4), without significant health risks, we can assume that the human gastric acid would be powerful enough to counteract the effects of venom compounds ingested by mother's milk and prevent their systemic absorption.

Having stated these facts, this should by no means imply that clinical toxicologists are to encourage a nursing mother to continue breastfeeding without applying caution. In fact, coagulopathy and thrombocytopenia due to the injection of venom are frequently reported adverse effects (5) affecting the general health of the mother. Moreover, the hematoma that would probably form following a snakebite would lead to breast pain and lactation failure. Therefore, we believe it seems wiser to make a decision based on laboratory data obtained for the mother. In the case of coagulopathy or thrombocytopenia, breastfeeding can resume after treatment with an antivenom and after coagulation tests and thrombocyte blood counts return to normal levels.

Nevertheless, it seems that properly designed studies need to be conducted on this important topic before any firm recommendation is made.

\section{REFERENCES}

1. Chippaux JP. Impact of the environment on envenomation incidence and severity. Med Sci (Paris). 2009;25:858-62. doi: 10.1051/medsci/20092510858. [Article in French]

2. Vonk FJ, Jackson K, Doley R, Madaras F, Mirtschin PJ, Vidal N. Snake venom: From fieldwork to the clinic: Recent insights into snake biology, together with new technology allowing high-throughput screening of venom, bring new hope for drug discovery. Bioessays 2011;33:269-79. doi: 10.1002/bies.201000117.

3. Dupre M, Gilquin B, Fenaille F, Feraudet-Tarisse C, Dano J, Ferro M, Simon S, Junot C, Brun V, Becher F. Multiplex quantification of protein toxins in human biofluids and food matrices using immunoextraction and high-resolution targeted mass spectrometry. Anal Chem 2015;87:8473-80. doi: 10.1021/acs.analchem.5b01900.

4. Liao J, Chao Z, Zhang L. Identification of common medicinal snakes in medicated liquor of Guangdong by COI barcode sequence. Zhong Yao Cai 2013;36:1740-2. PMID: 24956810 [Article in Chinese]

5. Kim JS, Yang JW, Kim MS, Han ST, Kim BR, Shin MS, Lee JI, Han BG, Choi SO. Coagulopathy in patients who experience snakebite. Korean J Intern Med 2008;23:94-9. doi: $10.3904 / \mathrm{kjim} .2008 .23 .2 .94$

Correspondence to: Dr Sayed Mahdi Marashi, Clinical Neurology Research Center, Medical School, Emergency Room/Division of Medical Toxicology, Hazrat Ali-Asghar (p) Hospital, Shiraz University of Medical Sciences, Shiraz, Iran. E-mail: marashi@sums.ac.ir 